\title{
LA ENSEÑANZA DE LA ATENCIÓN FARMACÉUTICA EN PREGRADO: LA EXPERIENCIA DE UNA UNIVERSIDAD DE LOS EEUU DE AMÉRICA
}

\author{
LILLIAN ARCE M. I
}

\begin{abstract}
RESUMEN
La práctica de Farmacia continúa evolucionando de su función tradicional de dispensación de medicamentos hacia una más especializada. Esta modalidad enfatiza el manejo y utilización de productos farmacéuticos dirigidos al cuidado del paciente, en el cual se paga por los servicios del cuidado farmacéutico. El propósito del curso «Patient Care Management» (PCM) es proveer al estudiante la oportunidad de practicar e integrar todos los aspectos correspondientes al cuidado farmacéutico. Por dos semestres consecutivos, los estudiantes que cursan el tercer año de Farmacia en los diferentes centros de enseñanza a distancia y en el campus central universitario, toman el curso que combina la clase y el laboratorio de práctica. Estos cursos se componen de una hora de clase por semana y tres horas de laboratorio, alternando semanas para un total de quince semanas durante el semestre de otoño (agosto-diciembre) y catorce semanas en el semestre de primavera (enero-mayo). En las clases, se repasan las 200 drogas más utilizadas, al presentar a los estudiantes casos de pacientes con diferentes estados de enfermedad basados en situaciones clínicas encontradas mayormente en la farmacia comunitaria. Las áreas que se cubren son: asma, hipertensión, diabetes, enfermedades del corazón, entre otras. Cada laboratorio cuenta con un promedio de tres farmacéuticos con experiencia, que tienen doctorado en Farmacia. Se asigna un promedio de 20-24 estudiantes por día de laboratorio, divididos en grupos de cuatro estudiantes por mesa de trabajo. El manual utilizado en el laboratorio titulado «Patient Care Management Lab» es requerido en cada sesión de laboratorio para un mejor aprendizaje y desarrollo del futuro Farmacéutico. Los estudiantes deben poder identificar problemas de terapia, hacer consejería al paciente, habilidad de poder interpretar recetas y reproducir etiquetas para los medicamentos dispensados al paciente. Los estudiantes tienen que exponer sobre dos fármacos de su preferencia ante la clase, enfocados al manejo de terapia, detección y evaluación de problemas relacionados al medicamento. Los estudiantes son «filmados» durante la consejería al paciente para auto evaluación. Este curso está diseñado para educar a los futuros farmacéuticos clínicos en relación a objetivos prácticos asociados con la práctica de farmacia del futuro, y a su vez lograr calidad y uniformidad en todos los centros de enseñanza (Ft. Lauderdale, West Palm Beach and Puerto Rico).
\end{abstract}

Palabras Clave: Atención Farmacéutica, Educación Farmacéutica

\section{SUMMARY}

The practice of Pharmacy continues to evolve from its traditional role of dispensing medication toward the management of use of pharmaceutical products in disease states, for which cost-effective pharmaceutical services are reimbursed. The goal of the Patient Care Management Courses is to provide the student the opportunity to integrate and practice all aspects of pharmaceutical care. For two semesters, third year students at local and distant sites take the courses that combine lectures and hands-on laboratory exercises. These courses consist of one-hour lecture per week and a three-hour laboratory on alternating weeks for the total of 15 weeks during the fall semester, and 14 weeks in the spring semester. Lectures review the top 200 drug products by presenting the students with disease-state based clinical situations encountered primarily in the community setting. Areas covered include diabetes, asthma, and hypertension, among others. Each laboratory session is staffed with three experienced clinical pharmacists, and 20-24 students divided into groups of four per working table. The «Patient Care Management Lab» manual is required for laboratory sessions. Students are assessed for identification of therapeutic problems, patient counseling, and tasks such as interpreting prescriptions and typing labels. In preparation for future in-service presentations, each student present two assigned drugs in class, focusing on management of drug therapy, detection and evaluation of medication-related problems. Students are videotaped while they counsel "patients" for selfevaluation. These courses, designed to educate our future clinicians about practical issues associated with current and future pharmacy practice, are standardized for uniformity among sites.

Key zoords: Pharmaceutical care, Pharmaceutical education

\section{INTRODUCCIÓN}

El termino Atención Farmacéutica fue definido en 1990 por Hepler y Strand, como la provisión responsable de la farmacoterapia con el propósito de lograr resultados que mejoren y prolonguen la calidad de vida del paciente ${ }^{(1,2)}$.

\footnotetext{
Department of Pharmaceutical and Administrative Sciences, College of Pharmacy, Nova Southeastern University. Florida, USA.
}

El farmacéutico del futuro tiene que tener la habilidad y el conocimiento para poder proveer información de la seguridad y eficacia de los medicamentos a pacientes, médicos y a todo el personal que esté envuelto en el cuidado de ese paciente. Al desarrollarse el concepto «Cuidado farmacéutico al Paciente" en los años 90 , no se suponía que revolucionaria la posición del farmacéutico tradicional, del despacho de medicamentos a uno, donde está directamente envuelto en el manejo de productos medicinales y el estado de enfermedad del pa- 
ciente. Al pasar los años, este concepto se ha refinado y diversos estudios han señalado que la intervención directa del farmacéutico a mejorado la salud, la calidad de vida y ha reducido los costos asociados con visitas a sala de emergencia, hospitalización de pacientes, e inclusive muertes ${ }^{(3)}$.

La misión y metas de la Facultad de Farmacia en la Universidad de Nova Southeastern (NSU) a través de años recientes ha estado enfocada a crear y desarrollar un currículo que satisfaga las demandas y necesidades de la profesión. El currículo requiere a los estudiantes llevar todos los cursos de ciencias básicas en el primer y segundo año. Para el curso: «Desarrollo del Manejo y Cuidado del Paciente (PCM)» los estudiantes tienen que haber completado Farmacodinamia, Farmacocinética y Terapéutica I. El curso PCM integra conocimientos aprendidos en años previos incluyendo despacho de receta médica, comunicación, leyes, consejería al paciente, farmacología y terapéutica entre otras. El propósito de que los estudiantes cursen PCM en el último año es que concurrentemente lleven Fisiopatología / Terapéutica II y III respectivamente es prepararlos para las rotaciones en hospitales e instituciones, además de entrenarlos para una práctica futura en farmacia de comunidad o en instituciones.

\section{DESCRIPCIÓN DEL CURSO}

El curso PCM se divide en dos semestres PHA 6710 y PHA 6720 e incluye dos partes: clase y laboratorio. La parte didáctica se enseña una hora por semana en semanas alternas y el laboratorio por tres horas durante ocho semanas alternas. Se cubren las 200 drogas más recetadas, el mecanismo de acción, los efectos secundarios, dosis entre otras. El laboratorio es interactivo, enfatiza el saber hacer y tomar decisiones e integra conocimientos y destrezas cubiertas en todos los cursos del currículo de Farmacia. Cada laboratorio cuenta con personal especializado, doctores en farmacia, que además de la función de enseñanza, traen al laboratorio experiencias personales de su área de práctica. En esencia lo que queremos lograr, es enseñar a los futuros farmacéuticos de nuestra facultad, a tener la habilidad y confianza de proveer al paciente un cuidado farmacéutico de primera.

\section{METAS Y OBJETIVOS DEL CURSO}

Este curso está diseñado para guiar a los estudiantes a desarrollar experiencia en el manejo, evaluación, comunicación, resolver problemas y práctica de farmacia en el escenario tradicional. Se espera que los estudiantes recuerden los puntos más importantes de las 200 drogas más recetadas, evalúen casos de pacientes del manual de trabajo, identifi- quen problemas y dar soluciones al problema, aprendan como enfocar y darle consejo al paciente acerca de las medicinas y productos sin receta. También se enseña como leer, procesar, interpretar, despachar, y administración de recetas médicas del mismo modo que aplicar guías legales y protocolos estandarizados de práctica clínica. Al terminar el curso el estudiante puede:

- Resolver problemas complejos y poder tomar decisiones éticas con relación a situaciones pro-. fesionales y sociales que integran valores personales y profesionales de la práctica de Farmacia.

- Tomar decisiones racionales, responsables cuando provea cuidado farmacéutico al paciente.

- Comunicarse adecuadamente con el público, pacientes, miembros del equipo médico, colegas y otros.

- Aconsejar y seguir de cerca al paciente con relación a usos y efectos de las medicinas de prescripción y no prescripción y terapias relacionadas incluyendo la promoción de la salud y el bienestar de la sociedad.

- Entender y demostrar los requisitos del sistema de distribución de varios escenarios de práctica, y leyes locales estatales y federales que regulan la práctica de farmacia.

- Analizar, interpretar y evaluar la literatura científica, la información dirigida al profesional y la literatura cotidiana comercial que llega a la farmacia, y poder proveer información y educación a pacientes, profesionales de la salud y público en general.

\section{INSTRUMENTOS DE ENSEÑANZA}

El manual utilizado en el laboratorio ha sido escrito y diseñado por el coordinador de curso ${ }^{(4)}$. El enfoque principal del manual es entrenar a los futuros farmacéuticos a interpretar las recetas médicas, por ser uno de los componentes básicos del cuidado farmacéutico. El cuidado del paciente también incluye conocer el perfil del paciente, estilos de vida, estados de enfermedad, género entre otras. Este manual ofrece la oportunidad a los estudiantes de realizar tareas requeridas con la dispensación de recetas presentadas en los perfiles de pacientes. Cada capítulo está organizado y corresponde a diferentes estados de salud que facilitan la Atención Farmacéutica (por ejemplo: hipertensión, enfermedad cardiovascular, geriatría, manejo del dolor, infecciones, diabetes, etc.). Los temas éticos y legales en el despacho de medicinas son considerados de igual modo que se toma en 
consideración las necesidades del paciente para mejorar el manejo de la enfermedad. El manual también provee al estudiante la oportunidad de practicar casos reales tomados de la vida real adaptados y facilitados por otros miembros del equipo de salud. Se provee a los estudiantes un ambiente seguro permitiéndoles ganar confianza y entendimiento en temas que envuelven manejo terapéutico de enfermedades crónicas que podrían involucrar sobre-utilización de los servicios de emergencia.

El propósito de usar perfiles de pacientes como medio de enseñanza es estimular en los estudiantes de Farmacia el pensamiento crítico que permita la identificación e interpretación de problemas, resolver problemas complejos, tomar decisiones importantes, racionales y responsables y la asimilación y difusión de información de medicamentos y equipo médico. Se evalúa en los estudiantes la comunicación y el uso efectivo de ella, cuando provee cuidado farmacéutico al paciente. Además se les presenta a los estudiantes escenarios de la vida real ocurridos diariamente en la farmacia de comunidad, como parte del entrenamiento para ayudarlo y promover el aprendizaje del futuro Farmacéutico.

\section{CONCLUSIÓN}

El curso Atención Farmacéutica fue desarrollado para adiestrar futuros farmacéuticos clínicos en un escenario que les permita continuar desarrollándose en el campo de esta nueva revolución de la Farmacia, que está enfocada al cuidado de salud del paciente donde el enfoque primordial de la práctica de farmacia es la Atención Farmacéutica. La atención farmacéutica comprende una combinación de consejería y consultoría entre paciente, médicos, miembros del grupo de salud y farmacéuticos donde el uso a propiado de fármacos y medicamentos de venta con receta son evaluados contra perfiles de pacientes y donde el estudiante es retado y forzado a desenvolverse activamente en la toma de decisiones para beneficio del paciente. Pacientes y consumidores en general esperan del Farmacéutico, que pueda proveer información adecuada relacionada a medicamentos venta con receta, sin receta, productos naturales, medicina complementaria, vitaminas, minerales e hierbas. Como profesionales de la salud, los farmacéuticos estamos conscientes que el uso inapropiado de medicamentos puede causar sufrimientos, aumentar el costo de salud, daño irreparable al paciente y hasta la muerte. El curso en la Facultad de Farmacia en NSU está diseñado para desarrollar profesionales que están siendo promovidos hacia una nue- va era donde la Atención Farmacéutica es un mejor servicio al paciente, los resultados son perfeccionados y la salud del paciente es una responsabilidad compartida con el grupo de salud incluyendo al paciente mismo. Según el sistema de salud continúe cambiando para beneficio del paciente los farmacéuticos tendrán que evolucionar también. En esencia, el enfoque del curso es preparar farmacéuticos en todos los aspectos de atención farmacéutica. Estos futuros farmacéuticos serán adiestrados a interpretar, procesar, dispensar y administrar recetas médicas del mismo modo que aplican directrices legales y criterios de práctica clínica.

\section{BIBLIOGRAFÍA}

1. Hepler CD and Strand LM. 1990. Opportunities and responsibilities in pharmaceutical care. American journal of hospital pharmacy [Am J Hosp Pharm] 47: $533-43$

2. Hepler CD. 1988. Unresolved issues in the future pharmacy. [Am J Hosp Pharm] 45: 107181

3. Kay B, Crowling G, Kershaw V, Robinson R. 1998. Perspectives on pharmacy's role in managed care American journal of healthsystem pharmacy [Am J Health Syst Pharm] 55: 1482-88.

4. Finkel R., Patient care management Lab: A workbook for Prescription Practice. 2002. 1rst. Ed. Florida, U.S.

5. Iinwood F. Tice, Am Institute of the History of Pharmacy 1976:241

6. Nau, D.P., Ried, L.D., Lipowski, E.E., Kimberlin, C, Pendergast, J., Spivey-Miller, S. 2000. Patients' perception of the benefits of pharmaceutical care. J Am Pharm Assoc 40 (1): 36-40

7. Bureau of Health Professions Report to Congress, «The Pharmacist workforce: a study of the supply and demand for pharmacists». Washington, DC: U.S. Department of Health and Human Services 2000.

8. Bootman et. al. 1997. The health care cost of drug-related morbidity and mortality in nursing facilities. Arch Intern Med 157: 2089. 96

9. Ernst FR, and Grizzle AJ. 2001. Drug-related morbidity and mortality: updating the cost-ofillness model. J. Am. Pharm. Assoc. 41: 192-9 